\title{
Effects of magnetic fields on capillary-gravity waves in the presence of magnetic surfactants
}

\author{
Alexander $V$. Zhukov ${ }^{1, *}$ \\ ${ }^{1}$ Institute of Mechanics, Moscow State University, 117192 Moscow, Russia
}

\begin{abstract}
The stability of capillary-gravity wave motion on horizontal free surface of viscous noncompressible fluid in the presence of magnetic surfactant in an external magnetic field was studied. It is shown that for normal as well as for tangential external magnetic field the horizontal free liquid surface is unstable for field strength exceeding some critical value that does not depend on the elastic constant of the surfactant film. However, for oblique external magnetic field the stability of the free surface depends not only on the field value but also on the surfactant elastic constant.
\end{abstract}

\section{Governing equations}

Recently, new surfactant molecules with magnetic properties have been synthesized [1]. This makes it possible the magnetic control over liquid surface properties by sufficiently strong $(0.4 \ldots 1 \mathrm{~T})$ external magnetic fields. The surface tension tensor for these media is anisotropic and depends on magnetic field strength. The analogous dependence was found for magnetic fluid - water interfaces subjected to moderate-intensity $(60 \ldots 110 \mathrm{G})$ magnetic fields [2]. We study the stability of capillary-gravity wave motion on horizontal free surface of viscous non-compressible fluid in the presence of magnetic surfactant in external magnetic field.

Let $D^{-}$be the domain occupied by liquid, $D^{+}$be the external domain and $\Sigma$ be the interfacial boundary, defined by the equation $z \equiv x^{3}=\eta\left(x^{a}\right), a=1,2$, where $x^{i}, i=1,2,3$ are the Cartesian coordinates: $D^{-}(t): z<\eta\left(x^{a}, t\right), D^{+}(t): z>\eta\left(x^{a}, t\right), \Sigma(t): z=\eta\left(x^{a}, t\right)$

In equilibrium $\eta=0$. The $z$ axis is directed upwards. The normal vector $\mathbf{n}$ on the boundary is defined as the external normal to $D^{-}$.

Inside the domain $D^{-}$the Navier-Stokes equations are valid:

$$
\begin{aligned}
& \partial_{k} v^{k}=0, \rho\left(\partial_{t} v^{i}+v^{k} \partial_{k} v^{i}\right)=\partial_{k} p^{i k(f)}+\rho g^{i} ; \\
& \mathbf{g}=(0,0,-g) ; p_{i k}^{(f)} \equiv-p \delta_{i k}+\mu\left(\nabla_{i} v_{k}+\nabla_{k} v_{i}\right)
\end{aligned}
$$

Here $\rho$ is the density and $\mu$ is the dynamic viscosity of the liquid, $v^{i}$ are the velocity components, $p$ is the pressure, $p_{i k}^{(f)}$ is the stress tensor (excluding the magnetic field stress) and $\mathbf{g}$ is the acceleration of gravity. Inside $D^{+} p=p_{0}=$ const .

The following conditions are valid at the interface [2]:

$\partial_{t} \eta-v^{3}+v^{a} \partial_{a} \eta=0$

$\left[H_{t a}\right]=-4 \pi \lambda_{n} \nabla_{a}\left\langle B_{n}\right\rangle,\left[B_{n}\right]=-4 \pi \lambda_{t} \nabla_{a}\left\langle H_{t}^{a}\right\rangle ;$

$\left[p_{b}^{i}\right] n_{i}-\nabla_{a} \sigma_{b}^{a}=0,\left[p^{i k} n_{i} n_{k}\right]-\sigma^{a b} b_{a b}=0$;

$p_{i k}=p_{i k}^{(f)}-\frac{H^{2}}{8 \pi} \delta_{i k}+\frac{H_{i} H_{k}}{4 \pi}$.

Here $\lambda_{n}=\Gamma b_{n}, \lambda_{t}=\Gamma b_{t}$, where $b_{n}, b_{t}$ are (generally different) constants defining surface magnetization of surfactant film in normal and tangential directions: $\mathbf{m}_{n}=-\lambda_{n}\left\langle\mathbf{B}_{n}\right\rangle, \mathbf{m}_{t}=-\lambda_{t}\left\langle\mathbf{H}_{t}\right\rangle[2], \Gamma$ is the surface surfactant density, $[f] \equiv f_{+}-f_{-},\langle f\rangle \equiv\left(f_{+}+f_{-}\right) / 2 ; \mathbf{H}, \mathbf{B}$ are the vectors representing magnetic field and magnetic flux density, respectively, $p_{i k}$ are the components of total stress tensor, $b_{a b}$ are the components of the second quadratic form of the boundary and $\sigma_{a b}$ are the components of surface tension tensor

$$
\begin{aligned}
& \sigma_{a b}=\left(\gamma_{0}-\beta \Gamma-\frac{1}{2} \lambda_{n}\left\langle B_{n}\right\rangle^{2}+\frac{1}{2} \lambda_{t} h_{c} h^{c}\right) a_{a b}-\lambda_{t} h_{a} h_{b}, \\
& h_{a} \equiv\left\langle H_{t a}\right\rangle
\end{aligned}
$$

The constant $\beta$ characterizes the dependence of surface tension on surfactant concentration [4]. The dependence of $\sigma_{a b}$ on magnetic field strength is taken from [2]. The following stability conditions are necessary: $\lambda_{n} \geq 0, \lambda_{t} \leq 0[3]$. 
The dynamics of surfactant surface density is described by the following equations:

$$
\partial_{t} \Gamma+\nabla_{a}\left(\Gamma u_{\Sigma}^{a}\right)+\frac{1}{2} \Gamma a^{a b} \partial_{t} a_{a b}=0 ; u_{\Sigma}^{a}=v_{t}^{a}
$$

Here the surfactant diffusion effects and mass transfer effects to or from the dividing surface are neglected and the surfactant surface velocity $u_{\Sigma}^{a}$ is taken equal to the tangential fluid velocity [4]; $a_{a b}$ are the components of first quadratic form on the surface.

The Maxwell equations for magnetic field in nonmagnetizable media are

$$
\operatorname{div} \mathbf{H}=0, \operatorname{rot} \mathbf{H}=0, \mathbf{B}=\mathbf{H}
$$

The conditions at the infinity take the form

$$
z \rightarrow-\infty: v^{k} \rightarrow 0 ; z \rightarrow \pm \infty: \mathbf{H} \rightarrow\left(H_{t}, 0, H_{n}\right)
$$

Here $H_{t}, H_{n}$ are the given values of external magnetic field strength in tangential and normal directions to the horizontal unperturbed surface.

\section{Linearization and dispersion equation}

To study capillary-gravity waves we perform the linearization of the equations (1)-(6) with respect to the equilibrium solution

$$
\begin{aligned}
& z>0: p=p_{0}, \mathbf{H}=\left(H_{t}, 0, H_{n}\right) \\
& z<0: p=p_{0}-\rho g z, v^{i}=0, \mathbf{H}=\left(H_{t}, 0, H_{n}\right) ; \eta=0
\end{aligned}
$$

Each of the perturbations may be represented as a superposition of normal modes, for example: $p^{\prime}=p-p_{(e q)}=\int \exp \left(s t+i k_{\alpha} x^{\alpha}\right) \hat{p}\left(k_{\alpha}, s, z\right) d^{2} k, \alpha=1,2$

Here $s=s\left(k_{1}, k_{2}\right)$ is the solution of the dispersion equation and the $(e q)$ subscript denotes values taken from the equilibrium solution (7).

The linearization of equations (1)-(6) give the following expressions for Fourier coefficients of the perturbations:

$$
\begin{aligned}
& \hat{v}_{3}=A \exp (k z)+C \exp (l z) ; l^{2}=k^{2}+\frac{s}{v}, \\
& v=\frac{\mu}{\rho}, \operatorname{Re}(l)>0 ; \hat{\eta}=\frac{A+C}{s} ; \\
& \hat{v}_{\alpha}=\frac{i k_{\alpha}}{k^{2}}(k A \exp (k z)+l C \exp (l z))+\varepsilon_{\alpha \beta} k^{\beta} B \exp (l z), \\
& \hat{p}=-\frac{\rho s A}{k} \exp (k z) ; \hat{\Gamma}=\frac{\Gamma}{s}(k A+l C) ; \\
& \hat{H}_{\alpha}=i k_{\alpha} \hat{h}^{ \pm} \exp (\mp k z), \hat{H}_{3}=\mp k \hat{h}^{ \pm} \exp (\mp k z) ; k=|\mathbf{k}| ; \\
& \hat{h}^{+}+\hat{h}^{-}=\frac{4 \pi b_{t} \Gamma H_{3} k \hat{\eta}}{1-2 \pi b_{t} \Gamma k}, \hat{h}^{+}-\hat{h}^{-}=-\frac{4 \pi i b_{n} \Gamma H_{\alpha} k^{\alpha} \hat{\eta}}{1-2 \pi b_{n} \Gamma k}
\end{aligned}
$$

Using these expressions we obtain the dispersion equation

$$
\begin{aligned}
& -\left(\omega_{0}^{2}+2 v k l s\right)\left(2 v k^{2} s+\varepsilon k^{3}-i \theta k^{3}\right)+ \\
& \left(s^{2}+\omega_{0}^{2}+2 v k^{2} s\right)\left(s^{2}+2 v k^{2} s+\varepsilon k^{2} l-i \theta k^{3}\right)=0
\end{aligned}
$$

where functions $l(k, s), \omega_{0}^{2}(\mathbf{k}), \theta(\mathbf{k})$ and the elastic constant of surfactant $\mathcal{E}[4]$ are defined as follows:

$$
\begin{aligned}
& \varepsilon \equiv \frac{\Gamma \beta}{\rho} ; l^{2}=k^{2}+\frac{s}{v}, v=\frac{\mu}{\rho}, \operatorname{Re}(l)>0 ; \\
& \theta(\mathbf{k}) \equiv \frac{1}{\rho}\left(\frac{b_{n} \Gamma}{1+2 \pi b_{n} \Gamma k}-\frac{b_{t} \Gamma}{1-2 \pi b_{t} \Gamma k}\right) \frac{H_{\alpha} k^{\alpha}}{k} H_{3} \\
& \frac{\rho \omega_{0}^{2}}{k}=\rho g+\left(\gamma_{0}-\beta \Gamma+\frac{\Gamma b_{t}}{2} H_{t}^{2}-\frac{\Gamma b_{n}}{2} H_{n}^{2}\right) k^{2}+ \\
& \left(\frac{\Gamma b_{n}}{1+2 \pi k \Gamma b_{n}}-\Gamma b_{t}\right)\left(H_{\alpha} k^{\alpha}\right)^{2}+\frac{\Gamma b_{t} H_{n}^{2} k^{2}}{1-2 \pi k \Gamma b_{t}}
\end{aligned}
$$

\section{Stability conditions}

If $H_{n} H_{t}=0$ then $\theta=0$ and the equation (8) is analogous to the dispersion equation for capillary-gravity waves in the presence of surfactant but in the absence of magnetic fields [4]; the only difference is the expression for $\omega_{0}^{2}$, that may be negative even for positive surface tension in the presence of magnetic fields. The stability condition in this case is

$$
\forall k_{1}, k_{2}: \omega_{0}^{2}(\mathbf{k})>0
$$

The condition (9) is equivalent to one of the more simple conditions $H_{n}<H_{*}$ or $H_{t}<H_{*}$, where $H_{*}$ is the critical value of magnetic field strength depending on other parameters of the problem. For $H_{n} \neq 0, H_{t}=0$ and three different values of $\delta=-\lambda_{t} / \lambda_{n}$ the dimensionless $q^{*}=\lambda_{n} H_{*}^{2} / 2\left(\gamma_{0}-\beta \Gamma\right)$ is shown on Fig.1.

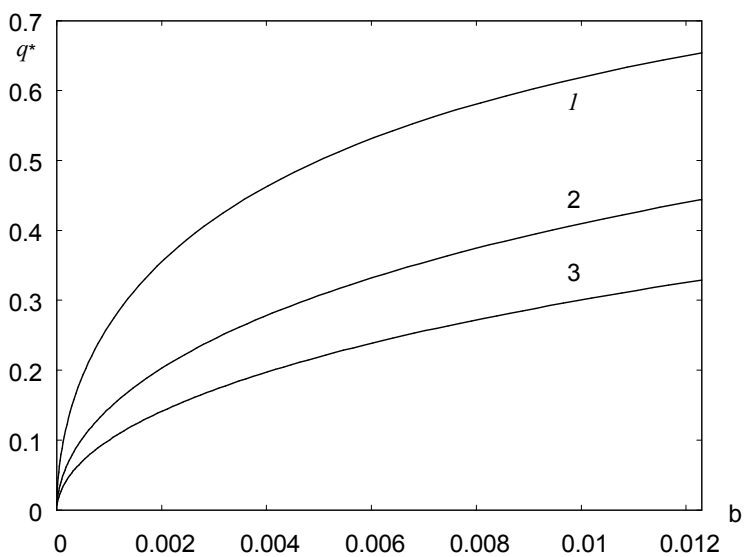

Fig.1. The dependence of critical dimensionless magnetic field strength $q^{*}=\lambda_{n} H_{*}^{2} / 2\left(\gamma_{0}-\beta \Gamma\right)$ on parameter $b=\left(\gamma_{0}-\beta \Gamma\right) / \rho g\left(2 \pi \lambda_{t}\right)^{2}$ at $\delta=0.1(1), 0.2(2), 0.3(3)$.

The function $q^{*}(b)$ is defined by the following parametric expressions:

$$
\delta q^{*}=t+2 t^{2}+t^{3}, b\left(1-q^{*}\right)=t^{2}+2 t^{3}, t>0
$$


For $H_{n}=0, H_{t} \neq 0$ the critical value of magnetic field strength is defined by the equation

$$
\gamma_{0}-\beta \Gamma+\Gamma b_{t} H_{*}^{2} / 2=0
$$

Note then the stability condition requires $b_{t}<0$ [3].The value of critical field may be estimated if we use the experimental data [1] qualitatively and suppose that for the magnetic field value $0.5 \mathrm{~T}$ the surface tension changes by $20 \%$. In this case the critical field equals $0.5 \sqrt{5} \approx 1.118 \mathrm{~T}$.

If $H_{n} H_{t} \neq 0$, the condition (9) is insufficient for the stability of the liquid surface. In that case the stability condition is equivalent to $\mathcal{E}>\mathcal{E}_{*}\left(H_{t} / H_{n}\right)$, where the critical value $\varepsilon_{*}$ depends on other parameters of the problem (see Fig.2).

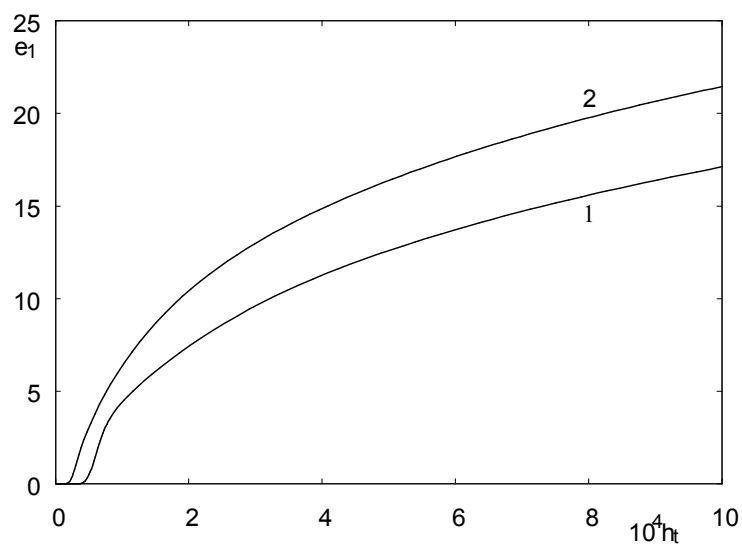

Fig.2. The dependence of $e_{1}=\varepsilon_{*} \rho / \sigma_{*}$ on dimensionless tangential component of external magnetic field $h_{t}\left(h_{t}^{2}=\lambda_{n} H_{t}^{2} / \sigma_{*}\right), \quad$ where $\sigma_{*}=\gamma_{0}-\beta \Gamma-\lambda_{n} H_{n}^{2} / 2+\lambda_{t} H_{t}^{2} / 2$, for the following parameters: $\lambda_{n} H_{n}^{2} / \sigma_{*}=1, v^{2} \rho / \sigma_{*} \lambda_{n}=0.1, \rho g \lambda_{n}^{2} / \sigma_{*}=10^{-6}$ and $\delta=0.1(1), \delta=1(2)$.

\section{The damping decrement}

The damping decrement of capillary-gravity waves depends significally on elastic constant. Let $s=S_{*}(\mathbf{k})$ be the solution of the dispersion equation with maximal $\operatorname{Re}(s)$, i.e. with minimal damping decrement. The dependence of $\operatorname{Re}(a) \equiv \operatorname{Re}\left(s_{*}\right) / \omega_{0}(\mathbf{k})$ on dimensionless wave number $m=v k^{2} / \omega_{0}(\mathbf{k})$ for several values of problem parameters is shown on Fig.3.

For small values of $e_{1} \neq 0 \quad \operatorname{Re}(a)<<1$ and $\operatorname{Re}(a) \rightarrow 0$ if $e_{1} \rightarrow 0$ but $e_{1} \neq 0$ and $k \neq 0$. Then, for sufficiently long wavelengths, the value of $\operatorname{Re}(a)$ increases with growing $e_{1}$ for sufficiently small $e_{1}<e_{1}^{*}(k)$ but decreases for $e_{1}>e_{1}^{*}(k)$ where $e_{1}^{*} \approx 0.3$.

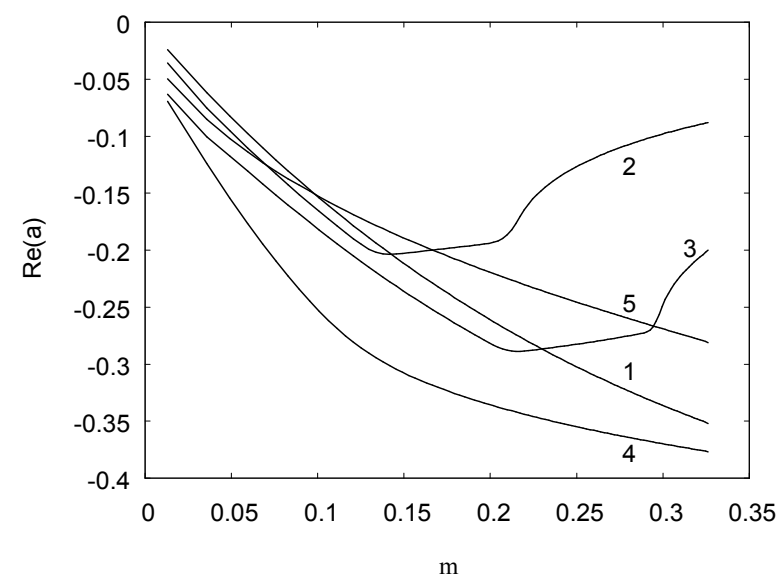

Fig.3 The dependence of damping decrement on dimensionless wave number for different values of dimensionless elastic constant $e_{1}=\varepsilon_{*} \rho / \sigma_{*}$ : $e_{1}=0(1) ; 0.05(2) ; 0.1(3) ; 0.3(4) ; 1(5)$. The main problem parameters are $\lambda_{t} / \lambda_{n}=-0.1, \lambda_{n} H_{n}^{2} /\left(\gamma_{0}-\beta \Gamma-\lambda_{n} H_{n}^{2} / 2\right)=1, H_{t}=0$.

\section{Conclusions}

The stability of capillary-gravity wave motion on horizontal free surface of viscous non-compressible fluid in the presence of magnetic surfactant in an external magnetic field was studied taking into account the anisotropy of surface tension tensor and the dependence of surface tension on surfactant surface density and on magnetic field strength. It is shown that for normal as well as for tangential external magnetic field the horizontal free liquid surface is unstable for field strength exceeding some critical value that does not depend on the elastic constant of the surfactant film. However, for oblique external magnetic field the stability of the free surface depends not only on the field value but also on the surfactant elastic constant.

The damping decrement of capillary-gravity waves depends significally on surfactant elastic constant.

Support by the Russian Foundation for Basic Research (projects Nos. 16-01-00157 and 17-01-00037) is acknowledged.

\section{References}

1. Brown P., Hatton T.A., Eastoe J. Current Opinion in Colloid \& Interface Science, 20, 140 (2015).

2. Golubyatnikov A. N., Subhankulov G. I. Magnetohydrodynamics (Engl. Transl.), 22, 62 (1986).

3. Golubyatnikov A. N., Uspekhi Mekhaniki, 4, 3(2006) (in Russian).

4. Levich V. G. Physicochemical hydrodynamics, Prentice-Hall, 1962, 700 pp. 